\title{
Atlas Construction for Dynamic (4D) PET Using Diffeomorphic Transformations
}

\author{
Marie Bieth ${ }^{1}$, Hervé Lombaert ${ }^{1}$, Andrew J. Reader ${ }^{2}$, and Kaleem Siddiqi ${ }^{1}$ \\ 1 School of Computer Science and Centre for Intelligent Machines, \\ McGill University, Canada \\ 2 Montreal Neurological Institute, McGill University, Canada
}

\begin{abstract}
A novel dynamic (4D) PET to PET image registration procedure is proposed and applied to multiple PET scans acquired with the high resolution research tomograph (HRRT), the highest resolution human brain PET scanner available in the world. By extending the recent diffeomorphic log-demons (DLD) method and applying it to multiple dynamic $\left[{ }^{11} \mathrm{C}\right]$ raclopride scans from the HRRT, an important step towards construction of a PET atlas of unprecedented quality for $\left[{ }^{11} \mathrm{C}\right]$ raclopride imaging of the human brain has been achieved. Accounting for the temporal dimension in PET data improves registration accuracy when compared to registration of $3 \mathrm{D}$ to $3 \mathrm{D}$ time-averaged PET images. The DLD approach was chosen for its ease in providing both an intensity and shape template, through iterative sequential pair-wise registrations with fast convergence. The proposed method is applicable to any PET radiotracer, providing 4D atlases with useful applications in high accuracy PET data simulations and automated PET image analysis.
\end{abstract}

\section{Introduction}

Medical image registration methods are necessary in a variety of clinical and research studies, whether it be aligning data between different subjects acquired with the same imaging modality (multi-subject single modality), or aligning data obtained from different modalities for the same subject (multi-modality single subject). The latter case is the most frequently used when dual-modality imaging is not available (e.g. to identify anatomical regions in PET images), and the challenge is to relate functional and structural images, e.g., PET to $\mathrm{CT}$ or PET to MR, as described in [6]. Such multi-modal registration is often limited to different images of the same subject. In the brain therefore, a rigid transformation can often be used, particularly when the acquisitions are close in time. However, the case of inter-subject single-modality registration requires more complex non-rigid methods to be deployed. Among these, Collins et al [3] develop a method for MR brain data that is now part of the MINC software suite. The Hammer algorithm [1] and the LDDMM framework [2] are also very popular for $3 \mathrm{D}$ to $3 \mathrm{D}$ non-rigid registration.

The problem of inter-subject PET to PET registration, however, is relatively unexplored in the medical imaging community. As an early example, the approach by Alpert et al. [1] and Eberl et al. uses only 6 parameters and cannot 
capture the necessary non-rigid deformations. In fact, there are no reported registration methods thus far which take into account the fact that PET data is intrinsically four dimensional and that much information is contained in the temporal dynamics of the tracer concentration. The currently used method for PET to PET registration is to use the sum of all frames of the scan as a single $3 \mathrm{D}$ image and then to apply a chosen $3 \mathrm{D}$ to $3 \mathrm{D}$ registration algorithm.

In this article we propose a fast algorithm for (4D) PET to PET inter-subject registration that uses information from the temporal behavior of the tracer to provide a diffeomorphic warping transformation between two datasets. We apply the algorithm to build a PET atlas of the human brain using a data set of 15 $\left[{ }^{11} \mathrm{C}\right]$ raclopride scans. The temporal behavior is regularized by tracer kinetic modeling to effectively reduce the dimensionality of the time course data. The algorithm is a natural extension of the DLD algorithm [12 which offers the advantage of being computationally efficient while sharing the potential for atlas building with other elegant mathematical frameworks such as LDDMM [2].

The applications of 4D PET radiotracer-specific atlases include their use as realistic ground truth radioactivity distributions in Monte Carlo PET simulations and furthermore they provide scope for far easier fully automated kineticmodeling of PET data, where it is necessary to identify key functional regions in the brain. A high quality atlas can first be carefully registered to an MR template, as a one off procedure, and then with relative ease a unique subject PET scan can be registered to the PET atlas for the matching radiotracer. A further application is to exploit radiotracer-specific atlases as spatiotemporal priors in both $3 \mathrm{D}$ and fully 4D image reconstruction, including the case of direct parametric image reconstruction requiring automated reference region identification, which could be again facilitated by a high quality PET atlas.

In the following section we present the extension of the DLD algorithm for PET to PET registration. We then evaluate the method in Section 3 and demonstrate its potential for building a PET atlas. We end with a discussion of this approach and its potential applications in Section 4.

\section{Methods}

In this section we review the 3D DLD algorithm, and then propose a new similarity measure for dynamic PET images, from which we develop an extension of DLD to dynamic (4D) PET images so that 4D PET atlases can be obtained.

\subsection{D Diffeomorphic Log-Demons Registration}

The DLD registration method was proposed by Vercauteren et al. 12 for 2D or 3D image registration. It uses the Lie group structure over diffeomorphisms for composition to obtain a diffeomorphic transformation by carrying out its computations in the log domain. The objective function to minimize is

$$
E(F, M, s, c)=\frac{1}{\sigma_{i}^{2}} \sum_{p}(F(p)-M(c(p)))^{2}+\frac{1}{\sigma_{x}^{2}} \operatorname{Dist}(s, c)^{2}+\frac{1}{\sigma_{T}^{2}} \operatorname{Reg}(s)
$$


where $p$ stands for position, $F$ is the fixed image, $M$ the moving image, $c$ the current non-regularized transformation, and $s$ the transformation, which is a regularized version of $c$. The first term is the similarity between the warped and the target image. The last term is the regularization term. The middle term is the distance between the regularized and the non-regularized transformation. By a first order approximation of $E$, the update $u$ to the transformation is

$$
u(p)=-\frac{F(p)-M \circ s(p)}{\left\|J^{p}\right\|^{2}+\frac{\sigma_{i}^{2}}{\sigma_{x}^{2}}} J^{p T} .
$$

More details about this method can be found in [12].

\subsection{TAC Based Similarity Measure}

A purely intensity based similarity measure is not appropriate for PET images, because they are sensitive to the exact dose of radiotracer injected. Therefore, we propose a new similarity measure for dynamic PET images that is based on the values of interest to researchers. We use the simplified reference tissue model (SRTM) proposed in [7, which is widely used by the PET community for binding potential computation. This approach models the circulation and binding of the injected radioligand in the brain. The time activity curve $C$ (TAC) at each voxel is then represented by three voxel specific parameters $\theta_{1}, \theta_{2}$ and $\theta_{3}$ and a spatially invariant reference TAC $C_{R}$, where the latter is estimated from a reference region in the image. For the case of $\left[{ }^{11} \mathrm{C}\right]$ Raclopride, the cerebellum is used. The relation between the parameters is as follows:

$$
C(p, t)=\theta_{1}(p) C_{R}(t)+\theta_{2}(p) C_{R}(t) * e^{-\theta_{3}(p) t}
$$

The least squares fitting of the model to the data is done by discretizing the search space for $\theta_{3}$ and employing standard optimization techniques for $\theta_{1}$ and $\theta_{2}$, according to the basis function method proposed by Gunn et al [5].

We propose to represent every image using the parameters $\theta_{1}, \theta_{2}$ and $\theta_{3}$, because they summarize all the relevant biological information. Therefore, an estimation of the parameters is done prior to the registration step using a $C_{R}$ estimated from the image, and a standardized $C_{R}$ is used for all the subjects to compute the similarity measure. The data are normalized in this fashion for inter-subject intensity variations and dose injection difference, while retaining biologically relevant and spatially varying information. Our new similarity measure can be expressed as

$$
\begin{aligned}
\operatorname{Sim}_{C_{R}}(F, M) & =\operatorname{Sim}_{C_{R}}\left(\theta_{1}^{F}, \theta_{2}^{F}, \theta_{3}^{F}, \theta_{1}^{M}, \theta_{2}^{M}, \theta_{3}^{M}\right) \\
& =\sum_{t=1 \ldots N} \sum_{p}\left(C^{F}(p, t)-C^{M}(p, t)\right)^{2}
\end{aligned}
$$

where $C$ is expressed for each image using Eq. 3. A standardized $C_{R}$ for the similarity measure can be obtained as an average of the reference TACs of several real scans. 


\subsection{Multi-frame Diffeomorphic Log-Demons Registration}

Similarly to the multichannel method [9], our extended method uses information from all individual frames. We extend the 3D DLD algorithm to 4D images with the assumption that all frames for a given subject are fully motion corrected. The transformation obtained is still 3D and must be applied to every frame of the image. The objective function from Eq. (1) is modified to

$$
E(F, M, s, c)=\frac{1}{\sigma_{i}^{2}} \operatorname{Sim}_{C_{R}}(F, M)+\frac{1}{\sigma_{x}^{2}} \operatorname{Dist}(s, c)^{2}+\frac{1}{\sigma_{T}^{2}} \operatorname{Reg}(s) .
$$

By setting derivatives with respect to $u$ of the first two terms of $E$ to zero, and following a similar process as in [12, it can be shown that the update u becomes:

$$
\forall p, u(p)=-\left(\frac{\sigma_{i}^{2}(p)}{\sigma_{x}^{2}} I+\sum_{t=1 \ldots N} J_{t}^{p T} J_{t}^{p}\right)^{-1} \sum_{t=1 \ldots N}\left(C^{F}(p, t)-C^{M}(s(p), t)\right) J_{t}^{p T} .
$$

The update computation requires a matrix of size $3 \times 3$ to be inverted at each voxel, with a computational cost that is acceptable. All computations are done in the log domain. Therefore, the warping transformation is guaranteed to be diffeomorphic. Moreover, as in the 3D method, the inverse transformation can be obtained at negligible computational cost by inverting the stationary velocity field. In our implementation, we use a multi-resolution approach.

\subsection{Building a PET Atlas}

A method proposed by Guimond et al [4] and widely used in atlas building is used along with our 4D DLD registration method to build a PET atlas. By iterating from an arbitrary initial reference image, this image is updated to move towards the barycentre of all images in the dataset. The term barycentre is to be understood as the Frechet barycentre of the warpings from each image to the reference. The Frechet mean can simply be expressed as the usual mean of the stationary velocity fields obtained from the Demons algorithm, as is done in 8 . The new reference image can be expressed as

$$
R^{\prime}=\left[\frac{1}{N} \sum_{i=1 \ldots N} I_{i} \circ \exp \left(v_{i}\right)\right] \circ \exp \left(-\frac{1}{N} \sum_{i=1 \ldots N} v_{i}\right)
$$

where $v_{i}$ is the stationary velocity field of the warping from $I_{i}$ to the current reference.

\section{Results}

The data used to test the registration algorithm and build the template are one hour $\left[{ }^{11} \mathrm{C}\right]$ Raclopride scans acquired on a Siemens High Resolution Research Tomograph (HRRT). All subjects were healthy volunteers. The size of each $4 \mathrm{D}$ image is $256 \times 256 \times 207 \times 26$, and each voxel is $1.21 \times 1.21 \times 1.21 \mathrm{~mm}^{3}$. More details related to the acquisition and processing of the data can be found in [10]. 


\subsection{Pairwise PET Registration}

We have compared our multi-frame extended demons algorithm to the classical 3D demons algorithm and to the minctracc software developed based on [3] , both applied on PET scans summed in time to yield 3D images. We compared the different methods in terms of intensity differences between corresponding voxel locations in pair-wise registrations.

Comparison of Minctracc and Multi-frame Demons. The minctracc software based on [3] provides a fully automated registration method. We used cross-correlation as the objective function (which in practice gave the best results in terms of minimizing voxel-wise differences in intensity). We note that this algorithm is not designed to be diffeomorphic and that it is primarily used for MR, so it is not a priori suited for PET. Our intention is to get a sense of how well an off-the-shelf 3D to 3D registration algorithm could work. We compared this method with the proposed approach on 5 subjects, i.e., on 20 pairs. Table 1 shows that the multi-frame demons method results in a lower percentage difference of intensity between the warped and the target image than the minctracc software, although it is highly important to note that even if a perfect registration were even possible, a large percentage difference between intensities would still be obtained due to variations in the unique radiotracer dose delivered to each subject as well as the unique physiology of each subject. There is an average decrease of intensity difference of 15 to 50 percentage points achieved by the proposed method compared to minctracc. This improvement can be attributed in part to the lack of explicit consideration of the temporal dimension in minctracc.

Comparison of 3D and Multi-frame Demons. We now compare the 3D and extended demons registration approaches on a dataset of 10 subjects (a total of $10 \times 9=90$ pairs) using the same regularization parameters for both methods. This gives us an indication of the potential improvement possible by incorporating the fourth (temporal) dimension in the registration process.

Table 2 shows the average percentage difference in intensity of the voxel values between the target and the warped image for 5 selected subjects from the dataset.

Table 1. Average percentage difference in intensity between voxels at corresponding locations in the target and the warped image for 5 subjects. Each box is for one pair of subjects and there are 20 pairs in total.

\begin{tabular}{ccccc||ccccc}
\multicolumn{4}{c||}{ Multi-frames demons (\%) } & \multicolumn{5}{c}{ Minctracc software (\%) } \\
- & 64.6 & 58.4 & 52.4 & 65.4 & - & 86.3 & 86.9 & 105.5 & 108.7 \\
45.8 & - & 44.4 & 43.9 & 39.8 & 65.7 & - & 59.8 & 71.6 & 80.4 \\
43.3 & 45.0 & - & 43.5 & 41.1 & 73.1 & 66.9 & - & 86.9 & 95.5 \\
45.9 & 53.3 & 51.7 & - & 54.4 & 83.2 & 79.2 & 82.1 & - & 90.8 \\
46.9 & 40.8 & 39.3 & 44.8 & - & 67.0 & 103.0 & 69.7 & 71.2 & -
\end{tabular}


Table 2. Average percentage difference in intensity between voxels at corresponding locations in the target and the warped image for 5 subjects. 20 pairs are shown in the table and there are 90 pairs in total.

\begin{tabular}{ccccc||ccccc}
\multicolumn{4}{c||}{ Multi-frames demons (\%) } & \multicolumn{5}{c}{3 D demons $(\%)$} \\
- & 64.6 & 58.4 & 52.4 & 65.4 & - & 75.5 & 71.0 & 59.7 & 76.2 \\
45.8 & - & 44.4 & 43.9 & 39.8 & 49.4 & - & 49.4 & 47.9 & 46.5 \\
43.3 & 45.0 & - & 43.5 & 41.1 & 47.7 & 51.3 & - & 48.0 & 44.8 \\
45.9 & 53.3 & 51.7 & - & 54.4 & 50.7 & 65.4 & 62.1 & - & 63.4 \\
46.9 & 40.8 & 39.3 & 44.8 & - & 50.4 & 47.3 & 43.9 & 48.6 & -
\end{tabular}

Over the whole dataset, a reduction of 3 to 12 percentage points is achieved by the proposed multi-frame method. Fig. 1 shows an example of registration were the multi-frame method is able to better recover structures of the target image than the $3 \mathrm{D}$ version.

We have observed that the magnitude of the determinant of the Jacobian of the transformation is smaller for the 3D DLD method, suggesting that it gets more easily trapped in a local minimum. The multi-frame method uses more information, which likely yields an objective function with fewer minima, allowing for larger deformations with a reduction in voxel intensity differences.

\section{$3.2 \quad$ A Template for PET}

The 4D template was built from $15\left[{ }^{11} \mathrm{C}\right]$ raclopride scans. Only three iterations of the algorithm were used because convergence was fast. Fig. 2 shows different frames and slices of the template. The blood vessels are clearly visible in the early frames, followed by the grey matter, the caudate and putamen. In the following frames, as expected, the radioactive concentration in the grey matter decreases quickly, while the radiotracer binds to the D2/D3 receptors of the caudate and putamen. The comparison with the single subject shown in Fig. 3 demonstrates how the template is far less noisy and has sharper edges.

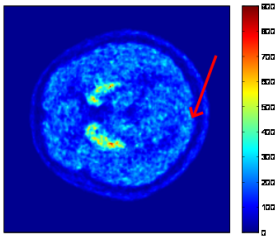

Target image

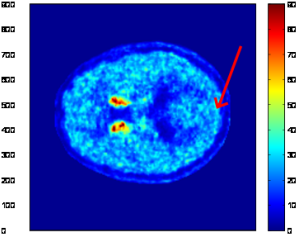

Source image

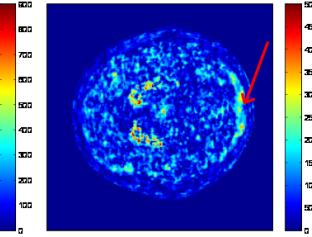

3D DLD

- Target image

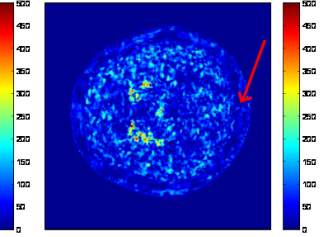

Multi-frame

- Target image

Fig. 1. Example of a registration. From left to right: target image, source image, absolute value of the difference of intensity between 3D DLD registration and target image, absolute value of the difference of intensity between multi-frame registration and target image. The red arrow shows a grey matter structure that the multi-frame registration recovers better than the $3 \mathrm{D}$ DLD registration. 

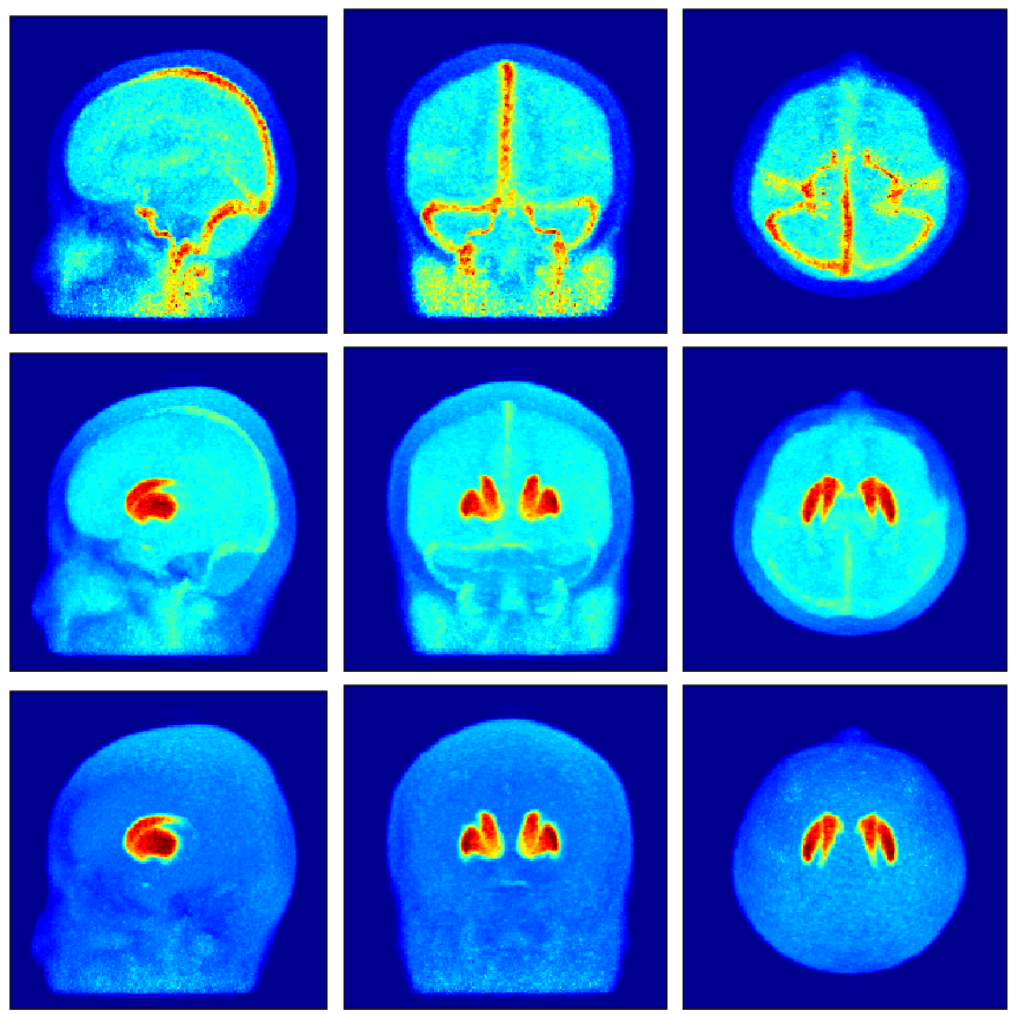

Fig. 2. Coronal, sagital and transverse maximum intensity projections of the 4D $\left[{ }^{11} \mathrm{C}\right]$ Raclopride atlas. First Row: frame 3 in the temporal sequence. Second Row: frame 12 in the temporal sequence. Third Row: frame 22 in the temporal sequence.
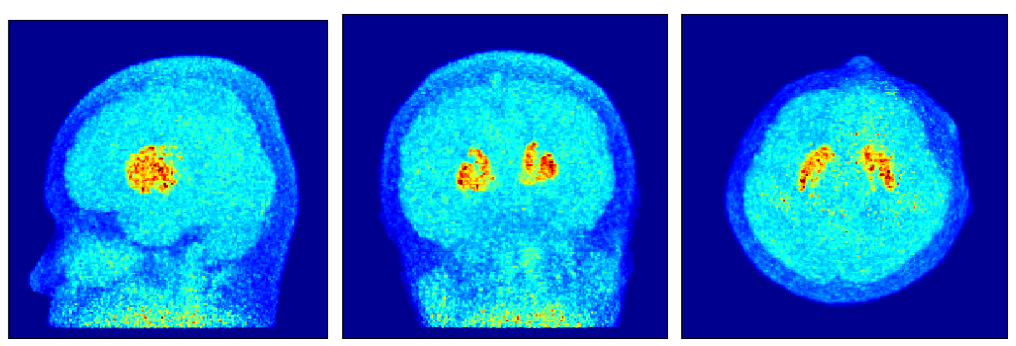

Fig. 3. Coronal, sagital and tranverse maximum intensity projections of a single $\left.{ }^{[1} \mathrm{C}\right]$ Raclopride subject, frame 12 in the temporal sequence. This is included for visual comparison with the template in Fig. 2.

\section{Discussion}

We have developed a new method for inter-subject dynamic (4D) PET image registration, based on an extension of the recent DLD method. Our method 
outperforms two 3D registration methods we have compared it against in terms of intensity difference. It also appears to be more resistant to local minima. By applying it initially to 15 dynamic $\left[{ }^{11} \mathrm{C}\right]$ raclopride scans from the HRRT, which is the highest resolution human brain PET scanner available in the world, we have taken an important step towards constructing a PET atlas of unprecedented quality for $\left[{ }^{11} \mathrm{C}\right]$ raclopride imaging of the human brain. The DLD approach was chosen for its ease in providing both an intensity and shape-based template. The proposed method is in principle applicable to any PET radiotracer, providing 4D atlases which will find useful application in high accuracy PET data Monte Carlo simulations as well as for automated PET image analysis. Furthermore, when used with appropriate care, such atlases could provide spatiotemporal priors for $3 \mathrm{D}$ and fully 4D PET image reconstruction.

Acknowledgments. The authors would like to thank Alain Dagher for helpful discussions and data and NSERC and FQRNT for research funding.

\section{References}

1. Alpert, N., Berdichevsky, D., Levin, Z., Morris, E., Fischman, A.J.: Improved methods for image registration. NeuroImage 3 (1996)

2. Beg, M., Miller, M., Trouvé, A., Younes, L.: Computing large deformation metric mappings via geodesic flows of diffeomorphisms. IJCV 61 (2005)

3. Collins, D., Neelin, P., Peters, T., Evans, A.: Automatic 3D intersubject registration of MR volumetric data in standardized talairach space. Journal of Computer Assisted Tomography 18 (1994)

4. Guimond, A., Meunier, J., Thirion, J.-P.: Automatic computation of average brain models. In: Wells, W.M., Colchester, A.C.F., Delp, S.L. (eds.) MICCAI 1998. LNCS, vol. 1496, pp. 631-640. Springer, Heidelberg (1998)

5. Gunn, R., Lammertsma, A., Hume, S., Cunningham, V.: Parametric imaging of ligand-receptor binding in PET using a simplified reference region model. Neuroimage 6 (1997)

6. Hill, D., Batchelor, P., Holden, M., Hawkes, D.: Medical image registration. PMB 46 (2001)

7. Lammertsma, A., Hume, S.: Simplified reference tissue model for PET receptor studies. Neuroimage 4 (1996)

8. Lombaert, H., Peyrat, J., Croisille, P., Rapacchi, S., Fanton, L., Cheriet, F., Clarysse, P., Magnin, I., Delingette, H., Ayache, N.: Human atlas of the cardiac fiber architecture: study on a healthy population. TMI 31 (2012)

9. Peyrat, J.M., Delingette, H., Sermesant, M., Xu, C., Ayache, N.: Registration of 4D cardiac CT sequences under trajectory constraints with multichannel diffeomorphic demons. TMI 29 (2010)

10. Qin, P., Duncan, N., Wiebking, C., Gravel, P., Lyttelton, O., Hayes, D., et al.: GABAA receptors in visual and auditory cortex and neural activity changes during basic visual stimulation. Frontiers in Human Neuroscience 6 (2012)

11. Shen, D., Davatzikos, C.: Hammer: hierarchical attribute matching mechanism for elastic registration. TMI 21 (2002)

12. Vercauteren, T., Pennec, X., Perchant, A., Ayache, N.: Symmetric log-domain diffeomorphic registration: A demons-based approach. In: Metaxas, D., Axel, L., Fichtinger, G., Székely, G. (eds.) MICCAI 2008, Part I. LNCS, vol. 5241, pp. 754-761. Springer, Heidelberg (2008) 